\title{
Leadership - Management of Management
}

\author{
Zdenek Dytrt \\ Associate Professor at Tomas Bata and Pardubice University, Czech Republic \\ Kladska 232, 0003 Hradec Kralove, Czech Republic \\ Tel: 420-724-046-976Ｅ-mail: zdenek.dytrt@tiscali.cz
}

\begin{abstract}
Radomir Serek
$\mathrm{PhD}$ graduate of Tomas Bata University in Zlin, Czech Republic

CEET Service Partner Manager in Siemens Healthineers

Na strani 632, 74245 Fulnek, Czech Republic
\end{abstract}

Tel: 420-602-218-156 E-mail: radomir.serek@ volny.cz

\begin{abstract}
Received: January 13, 2020 Accepted: February 17, 2020 Published: February 25, 2020
doi:10.5296/ber.v10i1.16523ＵRL: https://doi.org/10.5296/ber.v10i1.16523
\end{abstract}

\begin{abstract}
The dynamic development of the world is characterized by changes of the current state, that is by innovations, which are supposed to bring desirable economic effects. However, unethical intentions could also deliver the results fast but in an undesirable way. Unethical goals are mostly based on quantitative values and do not respect the existence of qualitative values and cannot satisfy the needs of the whole society.

Therefore, it is desirable to apply responsible management which respects both, quantitative as well as qualitative values. Formation of the ethical environment is a long-term task which requires managers who act as ethical leaders according to the certain principles. Management aims to change the status quo - thus management is the management of the innovation process. Management of management (leadership) is an innovation of the management theory and practice. The converging strategic goals should be a company's Goodwill and Corporate Culture. To reach these goals it will be necessary a cooperation between the business and educational and scientific organizations, as well as respecting the rules of the innovation process which includes continuity, timeliness, complexity and consistency.
\end{abstract}

Keywords: Innovation, Leadership, Managerial Ethics, Responsible Management, Unity of Quantity and Quality 


\section{Introduction}

There are new challenges which the companies are not sufficiently prepared for. Needs of resources, compliance to regulations, requirements for social standards, an adequate growth of productivity and quality, these are all challenges which the companies need to cope with. Some companies and their managers may use the shortcuts in a form of corruption, clientelism or undesirable business and political activities. These activities may deliver the desired goals more quickly and easily, but they damage relationships and the business environment as such.

On the other hand, an ethical and systematic approach to the problem resolution includes a complexity and a maximum achievable knowledge of its impact and consequences. Effective decision-making requires to respect an integrity of the solution itself that consequently contribute to a relatively long lasting and effective outcome.

Decision-making represents an input as well as an output of human activity in the society. It deals with the problems which are a priority for an individual, or the society, and their needs and interests. It is manifested by decision-making processes in the area of theory and practice of management which are focused to deal with specific situations or problems. However, they often fail to respect the context of the problem and thus an effectiveness of the resolution might not be optimal. Therefore, it is important to manage and innovate the management itself so that it would bring the desirable and effective outcomes for the whole society.

\section{Effective Innovations for the Manager}

Quantitative management decisions are mainly based on quantitative information and ignores fully or partially a qualitative side as well as the impacts on the environment and society. On the other hand, desirable innovations, in this respect, require a complex approach with having regard to the qualitative side, which always should be a part of the solution. Qualitative values do not result only from the quality of the material or the manufacturing process itself, but from the added value (quality), which is earned by respecting the qualitative values during the decision-making processes. These added (ethical) values testify an importance of ethical theory to be applied in the practice and are elementary for the quality of innovations, which bring the benefits to the whole society.

The quality in the decision-making processes is represented and manifested by managerial ethics. Quantitative management, which addicts to the technology and quantifiable parameters, and does not respect a unity of quantity and quality, contributes to the rise and development of consumerism and consumerist society. Due to the limited resources on our planet, $\mathrm{CO} 2$ emissions and pollution as such, the society should strive to leave or diminish the addiction to the quantitative management and to find an appropriate balance between quantity and quality. So called unity of quantity and quality or qualitative management creates positive values not only for the inventor (author/applicator of the innovation) but also for the users of the innovation, as well as the other stakeholders, including the environment and a neutral pollution trace.

A protecting and supporting element for the innovations would be a mature legislation and a 
stable political environment based on democratic principles. Karel Capek (2012), Czech writer who introduced the word robot, said that the democracy is based primarily on moral values. Thus, an application of managerial ethics is also important for the transparent and integral collaboration between administrative organizations, political and regulatory institutions issuing and enforcing the new legislations in cooperation with the business and crafts representatives (e.g. business chambers). This cooperation is important for the strategic goals, transparency and accountability across the whole society and contributes to the efficiency of the innovation process. However, an appropriate level of protectionisms should be taken into consideration (for example a patent protection), in compare to the (mostly non-democratic) systems where the intellectual property is not protected, neither respected at all.

The ethical environment is formed in the long term and by the contribution of managers who act as leaders according to the following principles (Dytrt, 2015):

- unity of quantitative and qualitative management,

- managerial ethics - system of morality, competence, implementation of ethics,

- people are the bearers of the sustainable development of society,

- leadership concerns everyone who manage (regardless if the organizations or just themselves),

- the consequence of not respecting managerial ethics is a consumerist management,

- decisions need to be understood as a change of the current state (which is actually an innovation),

- management is the management of the innovation process,

- management of management (leadership) is an innovation of the management theory and practice,

- system approach benefits from and is based on the management synergies,

- externalities - side effects of human doings; management decisions need to respect the external relations and their consequences as a system,

- respect for the social aspect of management (people are a subject as well as an object of leadership),

- Goodwill (good name of the company),

- Corporate Culture - should be a strategic goal of business companies,

- stimulation - motivation (respecting their equilibrium),

- benefit from women's inborn dispositions (like higher level of empathy, attention to detail, patience etc.) - which is, not yet fully, disclosed opportunity to improve the quality of management (Dytrt et al., 2014), 
- respect of specific conditions - every managed object is unique,

- leadership - dealing with problems in context and investigating the causes and consequences.

Responsible management is schematically illustrated on the following Figure 1:

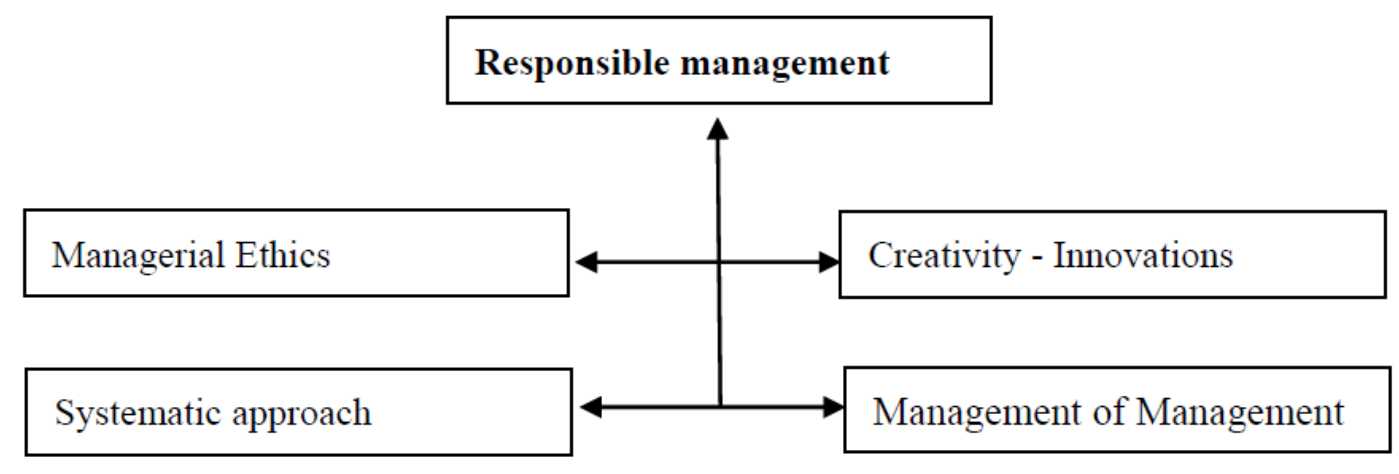

Figure 1. Responsible Management (own source, Dytrt, 2015)

The decision-making process should respect the dynamics of the society development, not only through the theoretical but also practical methods of management. Fields of science are narrowly focused and do not have ambitions to deal with other scientific fields and increase a complexity this way, which might be beneficial for innovation. The same applies also for the management theory which is more focused on management techniques and technologies rather than on the integration with other fields of science, which may enrich the management theory. Reversely the management theory may enrich also the other fields of science. Therefore, the lack of management integration leads to a loss for all involved parties.

The contemporary theory and practice of management is more focused on techniques, perhaps due to the technology achievements but also due to the high pressure on quantifiable outcomes. This only strengthens the quantitative management approach. However, the responsible and collaborative approach should also respect the qualitative side and complexity. These comprehensive information lead to the more effective decision-making as well as to the effective decisions themselves, which are an outcome of decision-making process - management.

Decisions, as the changes of the current state, are part of the innovation process and even could be its initiator. The innovation process itself might be decomposed to the partial innovations, in the similar way like the decision-making process can be decomposed to the individual partial decisions. A top manager decision (strategic) serves as an incentive for the lower management to induce partial innovations which forms (sums up) an incentive innovation.

Managerial ethics is a connection element for the individual production units and scientific areas and is an important mean to increase an integrity of the managed objects. The lack of complexity and integrity in the innovation process does not only lead to a reduction of the efficiency in the economic and social development, but also may lead to an increase of 
bureaucracy. On the other hand, managerial ethics increases the responsibility in decision-making processes and position the manager as an inventor (initiator of the incentive idea) and its early adopter and supporter.

Creation of innovations and their efficiency is in control of humans. The outcomes may be beneficial or opposite. Thus, it might be desirable to emphasize an importance of managerial ethics and even to think of its inclusion in the education systems.

Managerial ethics, respecting unity of quantity and quality, facilitates decision-making processes in order to support the invention and prevent subsequent problems right from the very beginning. In this respect, it is desirable to innovate the methods of education systems, not only within the frame of technology and techniques, but also within the frame of the theory and practice of management. The aim should be to raise awareness of the role and importance of management within human disciplines. It is about ethical decision-making and ethical thinking is about the content, form and goals of management in individual scientific areas. The graduates would be then supposed to apply these learnings in the practice. It does not matter how many human disciplines will apply this approach. More important is their cooperation, knowledge sharing and contribution to the whole society.

We are currently witnessing a debate on the question of human-being role in the future society. If and how will it be prepared to address the dynamics of hitherto unknown possibilities of technology, natural and social conditions. Would it be enough and reasonable to monitor the quantitative values and be a follower of their evolution only?

To what extent should one be responsible for the development of the whole society? There are methods in management theory that can provide a guidance to prepare the society for the future. It seems to be clear already now that the further intensification of the quantitative management will not address the challenges of the economy efficiency. It seems that these challenges will require men who will be ethical managers, not only of themselves, but also of the future society. Shall we get ready for the future? According to Stephen R. Covey, "Successful leaders will value principles more than new businesses" (Covey, 2009).

Respecting the unity of quantity and quality will increase the efficiency of decision-making process. Not respecting qualitative values will lead to not fully efficient decisions or to the situations which are not win (beneficial) for all involved parties. Quantitative management is more focused on win for one side (the side of the decision maker) and does not care much (or completely ignores) the other sides. This mostly leads to the arise of externalities. The outcomes of the quantitative management (based on non-complex information as a basis for decision-making) are mostly considered and addressed ex post - after the initial decision is applied. Therefore, it is desirable to promote "management of management" method which is originated in the humanist philosophy and is applied through the managerial ethics as part of its innovative theory. Its usefulness is demonstrated by the complexity of decision-making processes in management and other (scientific) activities.

\section{Rules of Innovation Process}

The innovation process has its own rules which, if not respected, reduce efficiency of the 
innovation. The innovation can have a form of a sine wave in both the positive and negative direction. In case of positive part, we speak about positive innovations, like achieving higher productivity or higher customer satisfaction or reduction of environmental pollution. Similarly, the negative part are the negative innovations, as for example a corruption, production failures, employee or customer dissatisfaction (Simson et al., 2006).

\subsection{Continuity}

Continuity concerns both positive and negative part of the sinusoid. It is divided into three parts (Dytrt, Striteska, 2009):

1. invention or a decision, which should be checked on its efficiency, whether its timely application have a desirable effect on the desirable changes of the inner or outer conditions. If yes, then a decision must be formalized in a form of an order towards the managers and workers. This is the first stage of innovation.

2. Time frame, in which the innovation fulfills its aim. It is considered as a boom which is specific to every innovation in the respect of time and level of action.

3. The impact of the innovation is not unlimited. Therefore, it is important to monitor the effectiveness of the innovation in time and develop new inventions, which would replace the current innovation when it becomes morally obsolete. The timing of the replacement is very important. If it would be too late or too early, then it could lead to the certain anarchy in the managed object.

\subsection{Timeliness}

The rule of timeliness is related to the rule of continuity with an accent on the timely creation and implementation of the innovation. It is not decisive the speed only but also if the innovation is implemented at the right point of time, else there is a risk of anarchy in the managed object but also that the market or society is not prepared for the revolutionary new concepts. More often the companies deal with the delay in admitting and implementing innovations, which may affect their competitiveness. Not only because they may launch the new products or services on the market too late but also because they may implement the innovations into their production processes too slow or not at all. This results in the loss of their competitiveness due to a stagnation of the productivity and high costs position.

Managers are to the high extent responsible not only for the creation, development and implementation of the innovations but also for their timing. Often, they fail to comply with the rule of timeliness. Either they hesitate too long with a decision to implement the innovation or (for example under a pressure of shareholders) they introduce the innovation too early. In both cases it is accompanied with the time of anarchy, which often leads to undesirable consequences. For example, a return of investment can be compromised. Or a definition of goals is not clear enough and employees are losing their orientation. Or the lack of preparedness of other internal and external factors which are supposed to facilitate the implementation of the innovation. This everything forms the certain anarchy in the managed object. The rule of timeliness aims to minimize the period of anarchy by preparing the new 
innovation already in the boom period of the still valid innovation.

Not only the preparation but also the exact timing, when the innovation should be realized, is critical. The early as well as the late implementation can bring the anarchy into the managed object. Thus, it is a responsibility and a challenge for the manager to decide about the right timing. Manager may rely on the opinions of the experts, as well as on the consultations with the research or other specialized institutions (Dytrt, Striteska, 2009).

\subsection{Complexity}

Non-complex approach to the projection, implementation and evaluation of the innovation usually brings lower effect than expected. Non-complex approach means to deal with the problems in an isolated way, without contemplating the consequences, root causes, relations and correlations with the other factors and so on.

Complexity of decision (order) can be achieved from the point of view of the overall impact on the managed object, which must be considered from both horizontal and vertical point of view:

a) horizontal point of view (action radius of the innovation/order), that is the area which is influenced by the innovation/order,

b) vertical point of view (action level of the innovation/order), that is to which extent the innovation changes the status quo.

The need of complexity is fulfilled if a given problem is analyzed from the both points of view, including the deeper individual approach to all possible problems and challenges as well as the relations among them, which may already exist or may appear over time.

Managerial practice tends to apply a routine decision-making based on the proven historical patterns. It is more comfortable for the managers themselves. They often ignore the new trends and findings in the scientific areas or apply them with a significant delay. Another example of managers' routine practice is their addiction to the measurable quantifiable performance indicators. These are easier to be collected and interpreted in compare to the qualitative indicators (Dytrt, Striteska, 2009).

Ignoring the complexity means to maintain the status quo with the routine management practices. Engaging complexity in the innovation management process means to create and think in a frame of an innovation network which should include the following areas:

- technology

- economy

- $\quad$ social (employees)

- legislation

- corporate responsibility 


\subsection{Consistency}

Fulfilling the rule of consistency provides a manager with the information about the efficiency of previous innovations and about the necessary or desirable precautions for the future innovation process. The way to ensure a rule of consistency is an analytic activity including measurable and non-measurable values that were reached by the functioning of managed system and what kind and level of economic and social benefit they bring. Computing and information technology play an important role in this analytic activity.

A success condition for the qualified and responsible strategic decision is to respect a complexity and synthesis, thus the unity of quality and quantity within the managed object. Important is also a consistent timing of preparation and realization of new innovations. Without that there is a risk of certain anarchy which may be accompanied with too early or too late implementation of the innovation (Dytrt, Striteska, 2009).

\section{Prerequisites for Applying Managerial Ethics}

The ability to create inventions and innovations is aligned with the ability to deal with the new state of things and relationships between them. The managers should have these prerequisites for applying managerial ethics (Dytrt, Striteska, 2009):

a) the ability to develop a courage to innovate and to take the responsibility and ownership for the inventions, and the new states and relationships they bring,

b) the ability to overcome the state of production or another activity in imagination, that is, the ability to imagine that things and relationships could be different than they are now,

c) the ability to develop the innovation and take the responsibility for it,

d) The manager cannot ignore the creativity of subordinates and degrade it to inactivity. Manager alone should not be the only bearer of the imagination and ingenuity, even though he is finally responsible for the innovations that have been realized. Usage of the employees' creative capabilities is the basis of the knowledge economy theory.

e) The corporate culture strategy, including the strategic objectives of scientific and educational institutions, allows to overcome the deepening of quantitative management.

The rule of complexity in decision-making processes reflects the maturity of management throughout its all phases, that means from the preparation of the decision to its final realization. It is the application of the innovation theory principles, which enable relatively balanced development of the factors that belong to the complexity of the addressed problems. Responsible management (Figure 2) should be respectful to the rules of innovation theory and managerial ethics, that is namely the consistency, comprehensiveness, timeliness and consistency. 


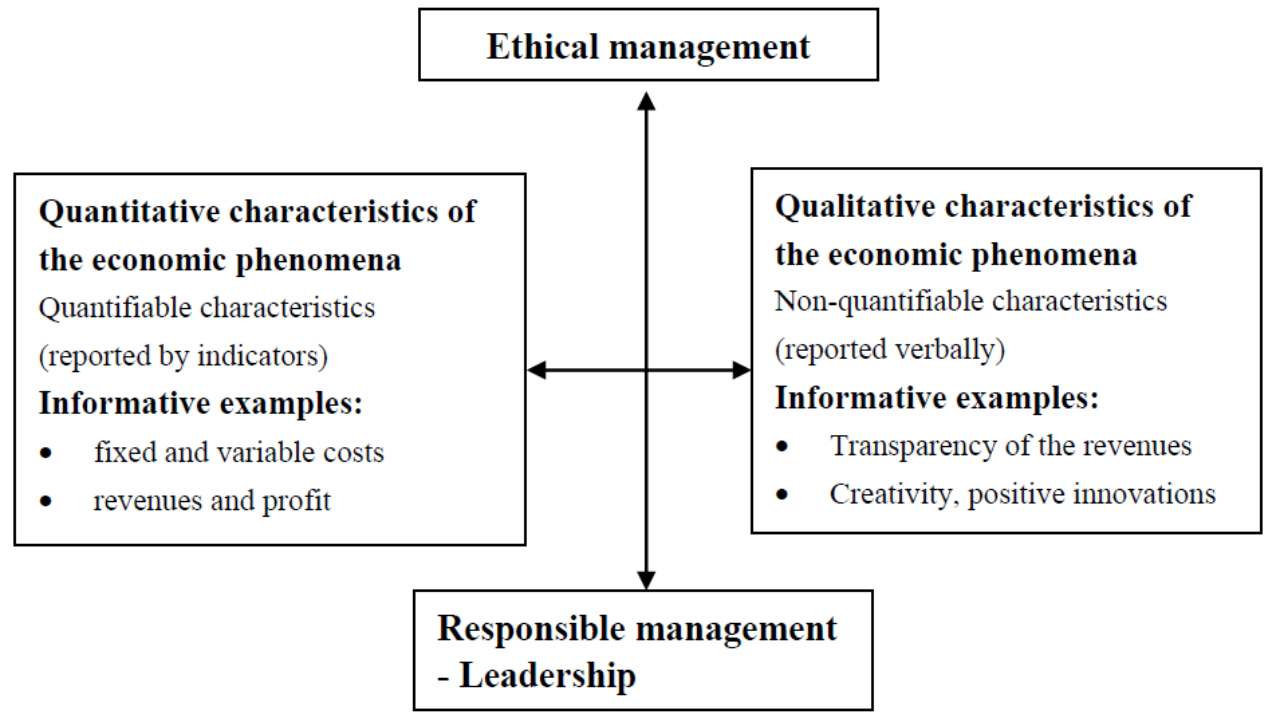

Figure 2. Responsible Management (Valenta, 2001)

Voice of top management should design the objectives and strategies comprising of economic, technical, administrative and social contexts. These should serve as triggers for the induced innovations by lower management levels, which bring their specific conditions into the process. This ensures the integrity of the subsystems and the complexity of the decision-making processes within the innovation management process. Creative teamwork is an enabling factor of decision-making processes. The more complex and costly the innovation, the more demanding and challenging is the innovation management process.

\section{Conclusion}

The current approach to the marketing management philosophy is focused on the creation of profit as a goal, and on its maximization while minimizing of the costs. Development boom in science and technology favored the use of techniques that facilitate and accelerate decision-making, which is a benefit for management. However, this kind of decision-making process is rather of a quantitative character because it is limited to measurable values that can be quantified. In decision-making processes, it is also necessary to embrace the qualitative aspects, which are non-quantifiable. Effective decisions may require considering both, quantitative as well as qualitative aspects, thus respecting of unity of quality and quantity. Non-adherence to this rule creates a potential for unethical decisions which does not lead to win-win situations.

The shareholder pressure on a rapid companies' growth has contributed to a creation of a quantitative management. On the other hand, the stakeholders' approach, which respects stakeholders' values and environmental aspects, has formed a need to innovate the management and apply managerial ethics and methods of leadership. This would guarantee that also the qualitative values will be respected within the decision-making. The philosophy of managerial ethics stands on a comprehensive assessment of the current state while utilizing a combination of both philosophical factors and the adherence to the unity of quantity and quality. This implies a need to overcome an individuality in the decision-making process 
(manager's approach) and to take an advantage of a cooperation (leadership approach).

It is a paradox of management that a manager is a subject and an object of the management at the same time. He should not expect to be handled in an ethical manner if he himself does not have a clear concept and the need of respecting the managerial ethics. If the manager does not demonstrate the ethical behavior in his actions, then he most probably will not succeed to persuade his co-workers to do so. Managers are visible and their actions serves like examples for others. However, they alone will not be able to change the whole society. It must start already in family and schools. Only this way a young man can earn the right attitudes to work and quality by following the right role models. The ethical leaders should cooperate with the educational institutions and should contribute to their educational programs. The future graduates should be then better prepared for developing of the society in the right direction and be able to deal with the questions and challenges related to the consumerism and consumerist society.

\section{References}

Capek, K. (2012). Hovory s T.G. Masarykem: Kniha apokryfu; O vecech obecnych, cili, Zoon politikon. Praha: Ceskoslovensky spisovatel, pp. 498.

Covey, S. R. (2009). Vedeni zalozene na principech. Praha: Management Press, pp. 302.

Dytrt, Z. (2015). Odpovedny management. Praha: Management Press. pp. 112.

Dytrt, Z., \& kol. (2014). Kvalitativni management - leadership: navrat do inovacni spolecnosti. Zilina: GEORG. p. 113.

Dytrt, Z., Gajduskova, A., \& Zadrazilova, D. (2014). Women and Management: Managerial ethics - qualitative management. Saarbrucken: LAP LAMBERT Academic Publishing. p. 188.

Dytrt, Z., \& Striteska, M. (2009). Efektivni inovace: odpovednost v managementu. Brno: Computer Press. pp. 160.

Simpson, P. M., Siguaw, J. A., \& Enz, C. A. (2006). Innovation orientation outcomes: The good and the bad. Journal of Business Research, 59(10-11), 1133-1141.

https://doi.org/10.1016/j.jbusres.2006.08.001

Valenta, F. (2001). Eticky aspect funkce manazera. In Z. Dytrt et al. Manazerska etika II inspirace pro 21. stoleti. Praha: Economia. pp. 92.

\section{Copyright Disclaimer}

Copyright for this article is retained by the author(s), with first publication rights granted to the journal.

This is an open-access article distributed under the terms and conditions of the Creative Commons Attribution license (http://creativecommons.org/licenses/by/3.0/). 